\title{
Evaluación educativa en educación parvularia: análisis de un modelo durante el proceso de enseñanza y aprendizaje
}

\section{Educational assessment in early childhood education: analysis of a model during the teaching and learning process}

\author{
Francisca Marina Toro Galaz
}

Universidad Finis Terrae

\section{RESUMEN}

Durante el proceso de enseñanza y aprendizaje en los niveles de educación parvularia es fundamental llevar a cabo una evaluación educativa sistemática, real, objetiva y en equipo, entendiéndola como una guía para el proceso. Es necesario comprenderla, además, como una instancia que favorece la observación, la identificación, el seguimiento y la toma de decisiones tanto para la mejora de la práctica educativa como para enriquecer el aprendizaje de los párvulos. La siguiente investigación-acción se llevó a cabo junto con los niños/as y el equipo de aula de un Nivel de Transición 2, y tuvo como objetivo mejorar las prácticas evaluativas, analizando el efecto de la evaluación en la construcción del aprendizaje de los párvulos. Para ello se 
ejecutaron y analizaron diversas intervenciones, las cuales demostraron, en los resultados, los efectos positivos de llevar a cabo una evaluación sistemática, no dicotómica y que se complementó con la autoevaluación de los niños/as.

Palabras clave: educación de la primera infancia, evaluación, enseñanza y formación, autoevaluación, análisis cualitativo

\begin{abstract}
During the teaching and learning process at the kindergarten levels, it is essential to carry out a systematic, real, objective, and team-based educational evaluation, understood as a guide for the process. It also needs to be understood as a body that encourages observation, identification, follow-up, and decision-making, both to improve educational practice and to enrich early childhood learning. The following research-action was carried out together with the children and the classroom team of Transition Level 2, and aimed to improve assessment practices, analyzing the effect of evaluation on the construction of learning in preschool children. For this, various interventions were executed and analyzed, which demonstrated, in the results, the positive effects of carrying out a systematic, non-dichotomous evaluation, plus a self-evaluation of children.
\end{abstract}

Keywords: early childhood education, evaluation, education and training, self-evaluation, qualitative analysis

\title{
INTRODUCCIÓN
}

Para afirmar en la práctica educativa con párvulos que un aprendizaje se ha logrado, este debe estar evaluado. Por ende, la evaluación se define principalmente como un proceso formativo, permanente y sistemático que permite reflexionar para tomar decisiones que nos lleven a la mejora pedagógica continua (MINEDUC, 2016). Una buena evaluación es el resultado de una planificación efectiva, con objetivos claramente definidos, capaz de monitorear procesos y avances de los niños y niñas, según lo establecen Letelier, Gómez y Vizcarra (2020). Debe centrarse así en el qué y cómo aprenden, 
ofreciéndoles las herramientas necesarias para que se hagan conscientes de la construcción de su propio aprendizaje.

A través de esta evidencia se determinó que los procesos evaluativos en educación infantil son determinantes; seguimos a Ibáńez (2020), para quien la evaluación educativa se enriquece con la participación de diversos agentes de la comunidad educativa, ya que cada uno recogerá elementos significativos que den cuenta de lo que realmente aprenden los niños y niñas. El objetivo de la evaluación es reunir la información pertinente para poner a disposición de los agentes educativos que componen el nivel, y que ofrezca una retroalimentación que motive progresivamente a los nińos/as a que tomen conciencia de sus procesos, logros, dificultades y formas de aprender (Díaz y Hernández, 2010).

Las investigaciones referidas a la evaluación en educación parvularia son escasas. Entre los referentes que se pueden encontrar destacan las de De la Garza (2004), Gómez y Seda (2008), Martínez y Rochera (2010) y Arrivas (2017), quienes establecen la importancia de analizar las prácticas evaluativas de las docentes y enfatizan la necesidad de llevar a cabo una evaluación formativa, que evidencie procesos y no solo resultados, es decir, que permita dar cuenta de los niveles de logro alcanzados por los niños y niñas.

\section{EVALUACIÓN EDUCATIVA EN EDUCACIÓN PARVULARIA}

Los procesos evaluativos en educación parvularia son determinantes según Rivera y García (2016), lo que se debe al acelerado crecimiento y desarrollo que se vive entre los 0 y 6 años. Durante este periodo -que es irrecuperable en términos biológicos y de receptividad neuronal-, los niños y niñas adquieren y desarrollan conocimientos, habilidades y emociones que son el anclaje de futuros aprendizajes (Battro, 2011; Jensen, 2004; Hoyuelos y Riera, 2015). 
Durante el contexto de la práctica profesional llaman la atención las observaciones a las instancias evaluativas desarrolladas por el equipo educativo del Nivel Transición Dos o Kínder -en adelante, NT2-; este equipo diseñaba e implementaba experiencias de aprendizaje en las que seleccionaban objetivos aleatorios, sin recurrir a información previa basada en los niveles de logro o en los intereses del grupo de niños y niñas. Esto hizo inferir que esas prácticas eran realizadas desde una perspectiva poco explícita con respecto al resultado de evaluaciones previas, que orientaran la toma de decisiones, de tal forma que se justificara determinada intervención. Por tal razón, se decidió efectuar esta investigación-acción junto al nivel educativo de un establecimiento ubicado en la comuna de Recoleta, en la Región Metropolitana, en Chile.

Se evidenció la ausencia de un respaldo de información sistemática y rigurosa respecto del proceso de enseñanza y aprendizaje, información que, según Rivera y García (2016), es necesaria para la evaluación educativa, ya que esta se utiliza como herramienta para fortalecer y guiar los procesos de evaluación en educación infantil porque permite visualizar de manera procesual los niveles de logro que alcanzan los niños/as, dejando evidencias que podrán fundamentar la selección de objetivos de aprendizaje posteriores.

Dos referentes técnicos esenciales que orientan la gestión pedagógica y el trabajo de aula en Chile determinan que la evaluación permite analizar y tomar decisiones respecto de las diversas líneas de acción, tales como aprendizaje, interacciones, tiempo, espacio, comunidad, entre otras. Estos documentos legales son herramientas técnicas diseñadas para facilitar en todo proceso de enseñanza-aprendizaje el desarrollo de competencias, la organización curricular, el desempeńo docente en el aula y el avance en las trayectorias profesionales y técnicas de los educadores; se trata de las Bases Curriculares para la Educación Parvularia (2018) -en adelante, BCEP- y el Marco para la Buena Enseñanza de Educación Parvularia (2019) -en adelante, MBE-EP-.

Las BCEP, en su calidad de currículo nacional, definen la evaluación como "una instancia formadora y una herramienta de apoyo al 
aprendizaje" (Subsecretaría de Educación Parvularia, 2018, p. 110). Desde esta perspectiva, este proceso se constituye como permanente, sistemático, reflexivo y dinámico, y permite, por una parte, seleccionar y construir progresivamente evidencia del proceso de aprendizaje y desarrollo integral de los párvulos y, por la otra, levantar un conjunto de acciones mediante la reflexión pedagógica en los equipos, con el objetivo de ajustar sus prácticas educativas y responder de manera pertinente y oportuna a las características, necesidades e intereses de todos los niños y niñas. En función de esto último, se consideró importante destacar la contribución de la evaluación para orientar la toma de decisiones consciente por parte del educador, ya que sin las evidencias no es posible realizar un análisis que conduzca a una toma de decisiones pedagógicamente fundada.

Respecto de lo establecido por el MBE-EP, que orienta a los educadores/as acerca de la reflexión y la práctica pedagógica que deben desarrollar como profesionales de la educación para la mejora continua de su quehacer:

Fija que la evaluación para el aprendizaje es esencial en el proceso de enseñanza y aprendizaje, lo que implica llevar a cabo evaluaciones sistemáticas que contemplen la diversidad de nińos/as, respetando ritmos y características particulares; siendo necesario definir distintas estrategias de evaluación como también instrumentos y medios de registro de información. Además, requiere realizar un análisis profundo de la información obtenida, lo que servirá posteriormente para retroalimentar tanto a los nińos y las niñas en sus procesos cotidianos, como también a las familias y equipos de aula. (Subsecretaría de Educación Parvularia, 2019, p. 21)

Sobre estas bases, se entiende que la evaluación en educación parvularia es principalmente formativa y formadora, es decir, también es una instancia para promover aprendizaje. Además, permite a los equipos pedagógicos reflexionar, construir y reconstruir experiencias de aprendizaje, dando mayor sentido y pertinencia a los procesos educativos que se desarrollan. Altimir (2011) establece que para llevar 
a cabo la evaluación se debe mantener una observación constante y focalizada, dando relevancia a un observador minucioso en el proceso de recolección de la información.

Desde otro punto de vista, la evaluación educativa es un proceso que también puede ser llevado a cabo en conjunto con los niños y nińas; esto gracias a la autoevaluación, que según lo establecido por la Subsecretaría de Educación Parvularia, se entiende como la valoración que realizan los niños y niñas de su propio aprendizaje y del proceso llevado a cabo para lograrlo (Subsecretaría de Educación Parvularia, 2020).

Este tipo de evaluación puede comenzar alrededor de los 3 años del niño o niña, edad a partir de la cual ya empiezan a manifestar las habilidades necesarias para la autovaloración, tales como el pensamiento reflexivo, la metacognición y la autorregulación. Y es un gran aporte tanto para el desarrollo personal de los párvulos, que enriquecen su autoconcepto y autonomía, como para el equipo educativo, que obtiene información explícita de la realidad del nivel educativo (Basurto et al., 2021).

El Sistema de Aseguramiento de la Calidad en Educación Parvularia (SAC), desde el 2020, implementa en los jardines infantiles que reciben aporte del Estado el Modelo de Evaluación y Orientación. Este consiste en un conjunto coordinado de componentes que evalúan, orientan e informan a los centros educativos y al Sistema de Aseguramiento de la Calidad, con el objetivo de propender al mejoramiento de la calidad y equidad de las oportunidades educativas en el nivel de Educación Parvularia. El Modelo de Evaluación y Orientación incluye cuatro componentes asociados a dos líneas de acción. La primera se enfoca en la evaluación y orientación del desempeño de los establecimientos educacionales, y tiene tres componentes: Diagnóstico Integral del Desempeño (DID), Priorización y Visitas de Evaluación y Orientación. La segunda se enfoca en la evaluación del desarrollo y aprendizaje de los niños y nińas e incorpora el componente Monitoreo Desarrollo/ Aprendizaje. 
En cuanto al DID, se establece que es un proceso de autodiagnóstico para la aplicación anual, que tiene como propósito contribuir al mejoramiento del desempeño de los Establecimientos de Educación Parvularia (EEP) en torno a las dimensiones de la gestión definidas en los Estándares Indicativos de Desempeño en Educación Parvularia (EID-EP) a través de la autoevaluación en el interior de las comunidades educativas (Subsecretaría de Educación Parvularia, 2020).

Para llevar a cabo este proceso, la Agencia puso a disposición de los establecimientos una plataforma digital con instrumentos que permiten el autodiagnóstico de la gestión, a través de una metodología participativa que incluye a distintos estamentos de la comunidad educativa.

Finalizado el proceso de autodiagnóstico, la comunidad educativa recibirá un reporte con la sistematización de los resultados obtenidos, en los que se especificará el nivel de logro alcanzado en las dimensiones de los EID-EP y las recomendaciones para la mejora.

\section{INVESTIGACIONES SOBRE EVALUACIÓN EN NIVELES NT2}

Se realizó una revisión bibliográfica con el objetivo de conocer estudios enfocados en la evaluación educativa de NT2 y se logró evidenciar que los procesos de aprendizaje en educación parvularia presentan referentes significativos, los cuales fundamentan metodologías activas y respetuosas desde el enfoque de derechos de niños y niñas, así como técnicas e instrumentos auténticos en evaluación que relevan la observación y documentación de la cultura infantil y su acompańamiento. En este sentido, los postulados de Malaguzzi (2001) y Hoyuelos y Riera (2015) destacan los procesos de aprendizaje y la evaluación como parte de ellos. Sin embargo, los estudios basados específicamente en los procesos de evaluación en NT2 son escasos, ya que las investigaciones en este nivel educativo se encuentran en 
su mayor parte relacionadas con los procesos de la lectoescritura y el pensamiento matemático (Muñoz, 2011; Norambuena et al., 2012).

En consecuencia, se infiere que el disminuido repertorio de evidencias sobre evaluación de este nivel podría estar influenciando las prácticas evaluativas que desarrollan los educadores/as, puesto que suelen ser enfocadas en los resultados, o incluso no realizarse. Sería beneficioso tener ejemplos y/o evidencias claras de lo enriquecedora que es una evaluación educativa que contemple diversos agentes, instrumentos y periodos, tal como lo declara el currículo nacional. De ahí que en esta investigación se buscó también aportar información que contribuya a investigaciones posteriores, tanto en el quehacer pedagógico como en los procesos de aprendizaje de los niños y niñas.

Es fundamental destacar que, durante la investigación, los diversos referentes concuerdan en el rol pedagógico de un educador/a, afirmando que este consiste en planificar la evaluación a largo, mediano y corto plazo, diseñando procesos evaluativos integrales, cooperativos, sistemáticos y pertinentes a la diversidad de todos los niños y las niñas, a las formas que tienen de aprender y a sus contextos de aprendizaje. Para ello, deben tener las herramientas necesarias para definir qué información es importante recoger para ajustar la práctica pedagógica con focos de observación, considerando la coherencia entre los objetivos de aprendizaje y las características de los niños/as del grupo (Subsecretaría de Educación Parvularia, 2020).

Por otra parte, debe establecerse también, junto con el equipo de aula, la forma de sistematizar y analizar la información recopilada, ya que se ha evidenciado que no siempre los registros facilitan la información específica (Ibáñez, 2020). En consecuencia, es necesario establecer estrategias, criterios y/o indicadores mediante los cuales se podrá observar cómo se despliegan los objetivos de aprendizaje (Paniagua y Palacios, 2005). De esta forma, la práctica reflexiva resulta eficaz para generar mejoras en el aula, prediciendo lo que se espera que ocurra con el aprendizaje, a la luz de ideas pedagógicas relevantes, como producto de una observación permanente e intencionada 
que permita evaluar y reflexionar para mejorar la acción educativa (Elmore, 2010).

\section{METODOLOGÍA}

La investigación se realizó en un establecimiento particular subvencionado de la comuna de Recoleta, Chile. Los participantes fueron el equipo educativo con una educadora, una asistente técnico, una educadora en formación, veinticinco niños/as y sus respectivos apoderados. El curso NT2 tiene un rango etario entre los cinco y seis años, edad que permitió responder al paradigma de investigación participativa con niñolas, construyendo herramientas que les facilitaron llevar a cabo una autoevaluación.

La investigación se realizó bajo el paradigma sociocrítico, dentro de la metodología cualitativa, la cual se orienta a profundizar casos específicos; su orientación no es prioritariamente medir, si no cualificar y describir el fenómeno social a partir de los rasgos determinantes, según sean percibidos por los mismos agentes que están involucrados en la situación estudiada (Bonilla y Rodríguez, 2005). Sumado a esto, según Santamaría (2013), este método cualitativo ha permitido una mayor y mejor comprensión de la realidad educativa, y ha causado un impacto positivo en el desarrollo de prácticas educativas contextualizadas, permitiendo una conexión entre teoría y práctica.

El tipo de investigación corresponde a una investigación-acción que tiene como propósito transformar la realidad (Elliott, 1993; Rojas 2012; Latorre, 2005) y que, a modo de síntesis, es una espiral de ciclos de investigación y acción constituidos por las fases: planificar, actuar, observar y reflexionar. Esto significa que dota de una mirada analítica y reflexiva sobre procesos colaborativos y reflexivos que favorecen la modificación de las prácticas sociales y pedagógicas. Los profesores "no mejorarán su enseńanza hasta que no cuenten con sistemas de interpretación y acción que les permitan vincular rigurosa y eficientemente las teorías con las prácticas” (Rojas, 2012, p. 8). 
Las diversas técnicas de recolección de información, que permitieron acercarnos a los diferentes informantes y, posteriormente, realizar un proceso de triangulación metodológica, fueron las siguientes:

Observación participante: Se realizó en todas las fases de investigación, durante todo el ańo 2019, en el aula de clases, reuniones de apoderados, etc. Tuvo diversos focos de observación basados en la necesidad de cada fase de la investigación, como se observa en la tabla 1.

Focos de observación según fases de la investigación

\begin{tabular}{ll}
\hline Fase de la investigación & Focos de observación \\
\hline Diagnóstica & Problemáticas del nivel \\
\hline Planificación & Intereses del nivel educativo y factibilidad de cambios \\
\hline Acción & Cambios realizados y posibles reestructuraciones \\
\hline Observación & Cambios establecidos para la mejora \\
\hline Reflexión & $\begin{array}{l}\text { Implicancias de los cambios realizados para la mejora de la } \\
\text { problemática y sus repercusiones }\end{array}$ \\
\hline
\end{tabular}

Fuente: Elaboración propia

Notas de campo: La investigadora realizó semanalmente notas de campo y tomas fotográficas en las fases de diagnóstico, planificación, acción y observación (Aguiar, 2015).

Entrevista grupal semiestructurada: Se preparó un guion temático con preguntas abiertas (Denzin y Lincoln, 2005) en las reuniones grupales de equipo que se realizaban una vez a la semana todos los miércoles, para construir en conjunto las acciones de mejora.

Encuesta: Serie de preguntas cortas a las familias y al equipo educativo con el objetivo de reunir datos o para detectar la opinión general (Guerrero, 2016). Se realizaron en la fase de diagnóstico con el propósito de conocer sus contextos; luego, en la fase de 
planificación, para construir acciones de mejora; y, por último, en la fase de finalización, con el objetivo de tener respuestas concretas y una valoración basada en la percepción de las acciones ejecutadas.

Para el levantamiento de los datos fue necesario considerar los aspectos éticos pertinentes. Tójar y Serrano (2000) hacen énfasis en que no todos los medios que en un momento dado están al alcance del investigador educativo son lícitos para alcanzar los fines de su estudio. Existe toda una serie de derechos que no deben ser quebrantados para no atentar contra la convivencia, la dignidad, la privacidad y la sensibilidad de las personas. Al mismo tiempo, la integridad de multitud de instituciones (centros educativos, hospitales, prisiones...), que pueden ser escenario de diferentes investigaciones educativas, debe ser preservada; particular atención merece la investigación educativa, ya que está en contacto con menores, familias, profesionales, entre otros.

En esta investigación, los principios éticos fueron respetados en las distintas fases del proceso investigativo, lo que tuvo implicaciones en la selección de las técnicas, el respeto al informante en cuanto a su privacidad y confidencialidad, el reconocimiento a sus aportes, el establecimiento de acuerdos y compromisos, y el consentimiento informado, el cual es definido por Garbin (2007) como la conformidad expresada por un individuo, con plena autonomía, para participar en una investigación. Se diseñaron consentimientos informados para participar en esta investigación, tanto como para el equipo de aula como para los niños y niñas pertenecientes al nivel educativo.

El objetivo principal de esta investigación en el aula fue mejorar las prácticas evaluativas en el proceso de enseńanza y aprendizaje junto al equipo del NT2, mediante análisis que evidencien su efecto en la construcción del aprendizaje de los niños y niñas. Para ello, se determinaron tres objetivos específicos: diagnosticar cómo se implementaba el proceso evaluativo en NT2 y cuál era su efecto en la atención a las necesidades e intereses de los niños/as; diseñar e implementar una propuesta evaluativa mejorada junto al equipo 
pedagógico, las familias y los niños/as; y, finalmente, reflexionar sobre el efecto de la propuesta evaluativa implementada en pro de visibilizar la importancia de la evaluación educativa sistemática y con diversos participantes. Con este propósito se realizaron diversas estrategias en cada fase de la investigación, generando cambios tanto en la organización del nivel educativo como en las prácticas evaluativas que se llevaban a cabo.

Por otra parte, para el trabajo con los niños y niñas se estableció una instancia de autoevaluación en las experiencias de aprendizaje. Así surgió la necesidad de diseñar un instrumento que facilitara y además invitara a reflexionar respecto de sus acciones, habilidades, desempeños, etc. Con este fin, se planificó una instancia de co-construcción con los niños y las niñas, de la cual surgió como instrumento el dibujo de dos vasos y la formulación de dos niveles de logro:

"Los niños y las niñas realizan una votación en la cual deciden que los niveles de logro serán: 'bien' y 'bien, pero podría mejorar', lo cual queda estipulado y será verbalizado posteriormente a las experiencias de aprendizaje. Sumado a esto, para representar sus autoevaluaciones gráficamente, se decidió crear el dibujo de un vaso al que se pintaron distintas cantidades de agua, según corresponda la autoevaluación" (Nota de campo, fase de acción).

Además, por medio de una comunicación, se invitó a los apoderados y apoderadas a participar en la evaluación del aprendizaje de los niños y niñas, presentándoles una escala de apreciación con simbología y espacio para registro, especificando explícitamente la acción a observar.

\section{RESULTADOS}

A lo largo de la investigación se realizaron análisis con el propósito de identificar la factibilidad de las mejoras, ya que este tipo de investigaciones permite retroceder y/o avanzar en el desarrollo de las fases. En cuanto a la fase de finalización, se desarrollaron análisis 
guiados por el proceso de triangulación metodológica, la cual, según lo establecido por García et al. (2016) es una estrategia de integración metodológica en la que se privilegia la utilización de distintos métodos. Vera y Villalón (2005) detallan que en el proceso de triangulación los resultados se organizan y analizan, y se fundamentan los datos obtenidos a lo largo de las fases que componen la investigación para la captura de un mismo objeto de la realidad social. Con esta estrategia se pretende, ante todo, "reforzar la validez de los resultados" (Bericat, 1998, p. 111), lo cual es posible mediante la convergencia o divergencia de los resultados obtenidos con cada uno de los métodos empleados. Esto, con el propósito de visualizar las implicancias de las intervenciones realizadas.

Los resultados que se presentan a continuación se desarrollan en cuatro grandes temas, ya que, para la ejecución de esta investigación se hizo una sistematización de categorías apriorísticas y emergentes (Elliot, 1993).

\section{Acciones de la evaluación e implicancias}

Durante la fase de diagnóstico, a medida que se utilizaba el método de observación participante y notas de campo en el establecimiento, se logró visualizar que la educadora del nivel no realizaba evaluaciones posteriores a su intervención pedagógica; por el contrario, se logró conocer que su método de trabajo consistía en rellenar una lista de cotejo cuando el tiempo alcanzaba. Ante la ausencia de sistematización de la información respecto de los objetivos de aprendizaje trabajados, se realizó una primera entrevista a la educadora y asistente técnico de la sala. Ellas respondieron:

"La evaluación es un proceso que permite visualizar el avance de los párvulos, por lo que se considera súper importante; pero hay ocasiones en que el tiempo no me alcanza, y lo más rápido es llenar una lista de cotejo donde además le pregunto a mi compañera si ha observado lo mismo. Por otro lado, desde la unidad técnico-pedagógica del establecimiento nos envían pruebas, las cuales nosotros aplicamos 
a los niños y niñas a nivel institucional para hacer entrega de algún informe a las familias o al Ministerio de Educación” (Educadora, entrevista, fase diagnóstica).

"Yo considero que es muy importante, ya que esta nos permite conocer las necesidades de los niños y nińas para reforzarlos en lo que están mayormente descendidos. Y sí, yo participo en la evaluación; a mí la educadora siempre me pregunta si estoy de acuerdo con lo que ella pone en los informes al hogar" (Asistente técnico, entrevista, fase diagnóstica).

$\mathrm{Al}$ analizar las respuestas en esta parte de la entrevista, se logró obtener como evidencia que el equipo de aula reconoce la importancia que tiene la evaluación. Sin embargo, tenían apreciaciones diferentes y desactualizadas respecto de lo que afirma el MBE-EP (2019) como prácticas evaluativas en los niveles de educación parvularia. Asimismo, se logra apreciar que estas acciones repercuten en la práctica con los niños/as, generando escasa participación de agentes evaluativos, experiencias de aprendizaje sin preparación y carencia de información sistematizada para el diseño de próximas intervenciones pedagógicas. A raíz de esto, se evidencia que esta categoría presenta divergencia, puesto que se observa una discrepancia entre la declaración realizada por el equipo educativo sobre la evaluación y lo que realizan en su práctica diaria.

Posteriormente se realizó una reunión para conversar con el equipo de aula respecto del análisis del resultado de la entrevista, logrando que surgieran algunas ideas acerca de posibles mejoras. La primera propuesta fue diseñar un instrumento evaluativo que permita situar en niveles de logro a los niños y niñas, pero también hacer registros descriptivos, tareas para las cuales se decidió en conjunto reemplazar la lista de cotejo por una escala de apreciación que tenía un ítem para registros descriptivos.

Se diseñó una tabla específicamente para el nivel; esta contemplaba lo necesario en una escala de apreciación que debieron rellenar con información abreviada especificada en una simbología, la cual 
describe exactamente lo que está señalando el agente evaluativo; además, incluye un espacio que les permitió comenzar a registrar las acciones relevantes que observaban en los niños y niñas a lo largo de sus procesos. Si bien este instrumento es una versión básica, para este contexto el hecho de incluir otro nivel y además la observación para tomar registros descriptivos representó un desafío para ellas y su organización diaria.

Durante una semana se observó y se analizó la factibilidad de la intervención realizada, que luego se comentó en la siguiente reunión de equipo, en la cual declararon que:

"Los cambios que hicimos fueron súper buenos para el nivel; nos sirvió tanto para tener el tiempo de evaluar como para conocer a los chiquillos de una manera más personalizada y conocer sus necesidades" (Educadora, nota de campo, fase de acción).

"Me gustaron los cambios porque no ha sido difícil adoptarlos, ya que en el establecimiento uno no tiene mucho tiempo con la rutina tan establecida, pero con los niños en grupos chicos es súper fácil sentarse con ellos e ir evaluando en la escala" (Asistente técnico, nota de campo, fase de acción).

Esta escala permitió que el equipo educativo estableciera un momento y un lugar físico para llevar a cabo la evaluación, favoreciendo tanto las interacciones entre el equipo como con los párvulos. Además, favoreció el análisis de la práctica educativa en las reuniones de equipo, reflexionando la información y proponiendo acciones de mejora.

\section{Ejecución de la evaluación}

Se evidenció que en el nivel educativo se llevaban a cabo como procesos de evaluación el relleno de un instrumento dicotómico llamado lista de cotejo y pruebas estandarizadas diseńadas desde la Unidad Técnico-Pedagógica -en adelante, UTP-, homogéneas a todos los NT2 del establecimiento. Por medio de una entrevista 
realizada al equipo de aula para recoger información relacionada con los cambios de mejora que podrían implementar en torno a la evaluación educativa, se constató que:

"Yo considero que un aspecto a mejorar de la evaluación es que sea más ordenada y personalizada, porque pasa que al momento de ir a sentarse con los chicos/as a evaluar y tomar registro de ellos, a pesar de tener un instrumento nuevo no se alcanza a evaluar a todos, teniendo en cuenta que son cuatro aprendizajes esperados a evaluar al día" (Educadora, encuesta, fase de planificación).

"Yo considero que las prácticas evaluativas, el día de hoy, sean más reales y personalizadas, donde se vean realmente los logros de los niños y niñas, ya que así será mucho más factible ayudarlos en lo que necesiten" (Asistente técnico, encuesta, fase de planificación).

"Diariamente, se realizaban cuatro experiencias de aprendizaje en el nivel educativo; la educadora daba inicio a las experiencias sin tener conversaciones previas con la asistente técnico, por lo que su aporte era desde atrás, acompańando a los nińos/as para mantener su atención o, en ocasiones, se mantenía al margen mientras revisaba las agendas; rara vez se acercaba a preguntar de manera autónoma qué se iba a trabajar o qué se pretendía evaluar" (Nota de campo, fase de planificación).

Las acciones implementadas se tradujeron en reestructuraciones tanto en el horario del nivel educativo como en la organización del aula, y determinaron la formación de grupos pequeños para favorecer los tiempos de evaluación. Por consiguiente, la mejora de esta organización permitió llevar a cabo una evaluación educativa con un instrumento guía que les permitió sistematizar la información y establecer los tiempos para el proceso de la evaluación, ya sea el de observación o el de análisis por parte del equipo.

No obstante, cuando se propuso el desarrollo de la investigación, la recepción fue negativa, ya que implicaba para ellas más trabajo del que tenían, lo cual nos invitó a generar estrategias para favorecer la 
motivación, tales como instancias de reflexión sobre nuestras prácticas y proyecciones de los posibles resultados. Por lo tanto, al comenzar la ejecución de las acciones diseñadas para mejorar la evaluación en el nivel, el equipo se manifestó con mucha disposición, permitiendo que se generara un clima positivo y que todo el nivel tuviera conocimiento sobre qué, cómo y cuándo se evaluaba.

"Tú crees que es posible evaluar tantos niños y niñas con registro? Eso es porque aún no has trabajado; cuando tengas tu sala verás lo complicado que es evaluar a todos" (Educadora, nota de campo, fase de planificación).

"Al comenzar el día, cada mañana el equipo se reúne en el aula unos minutos para conversar sobre la planificación, dejando explícitos los focos de observación que se tendrán durante el día para evaluar" (Nota de campo, fase de acción).

"Las adultas se sitúan en los grupos de trabajo siempre con la escala de apreciación y una hoja en blanco para registrar mientras observaban y realizaban preguntas respecto de las acciones que ellos realizaban, lo cual era guardado en una carpeta azul establecida como la carpeta de evaluación que tenía cada agente educativa” (Nota de campo, fase de acción).

De este modo, se reconoció la posibilidad de llevar a cabo una evaluación educativa sistemática y que esta puede ser adaptada a diversos contextos o interpretada por diversos agentes con el motivo de que enriquezca tanto la práctica educativa del equipo como los procesos de aprendizaje de los párvulos.

\section{Conocimientos sobre la evaluación en educación parvularia}

Respecto del conocimiento del equipo sobre los documentos establecidos por el Ministerio de Educación, ya sean las BCEP como el MBE-EP, los cuales están destinados a guiar el quehacer pedagógico bajo el 
currículum nacional, la educadora declara tener conocimiento, pero no evidencia un uso constante de esas directrices.
"Los conozco, y comprendo a lo que apuntan y además utilizamos algunos cuando se termina el año escolar, ya que se realiza una reunión donde todas las educadoras trabajan para crear o mejorar los diseños de experiencia de aprendizaje que quedarán listos para el proceso de diagnóstico del año siguiente. Para esto utilizamos las Bases Curriculares y, al mismo tiempo, hacemos una selección de indicadores con los hitos del desarrollo y mapas de progreso para establecer metas específicas para cada nivel educativo" (Educadora, encuesta, fase de diagnóstico).

En relación con este aspecto, en la fase de planificación se logró evidenciar que la información entregada es coherente con lo que ella realiza, ya que la educadora sustenta sus experiencias en lo establecido por las BCEP en todo momento. Sin embargo, algunas observaciones fueron claves para reconocer un desafío en el diseño de indicadores evaluativos.

"Cada dos semanas se hacía entrega de las planificaciones al jefe de UTP, quien se encargaba de revisarlas para corregirlas, devolverlas y luego recibirlas con los errores mejorados; en reiteradas ocasiones sucedió que él corregía los indicadores de evaluación haciendo énfasis en que debía analizar la habilidad que se quería potenciar, ya que solía crear indicadores que se alejaban de lo que planteaba el objetivo de aprendizaje" (Nota de campo, fase de diagnóstico).

Esto cambió después de la investigación, ya que sus diseños de experiencia se basaban en experiencias anteriores con objetivos e indicadores de aprendizaje ya trabajados que necesitaban ser potenciados según lo evidenciado a través de las evaluaciones previas.

Por otro lado, esta información era complementada según la sugerencia de la UTP por la taxonomía de Bloom, la cual (Losada y Iturbide, 2011) establece una jerarquía con seis niveles de grado 
creciente de aprendizaje del estudiante, que facilita diversos indicadores de habilidades basados en los niveles de logro.

\section{La co-construcción de la evaluación}

En la fase de diagnóstico se logró evidenciar que el proceso de evaluación era llevado a cabo solamente por la educadora, que en ocasiones le preguntaba a la asistente técnico si estaba de acuerdo con la información reportada. Por otro lado, estaba la evaluación diseñada desde la UTP, que también debía ser aplicada y revisada por la educadora del nivel. El equipo menciona, por medio de una entrevista, que la evaluación es realizada en conjunto.

"Yo realizo la evaluación según lo que he visto en los niños y niñas
observando los indicadores que se establecen a principios de año en
conjunto con las otras misses, pero la asistente también forma parte
de la evaluación, porque uno le pregunta si ella ha visto lo mismo
o si cree que es necesario poner algo diferente. Y en cuanto a las
familias, uno les comunica constantemente qué necesita mejorar
su hijo/a, por lo que sí son parte de la evaluación" (Educadora,
entrevista, fase diagnóstica).

"La educadora del nivel, siempre que realiza la evaluación, me la muestra y me pregunta qué me parece; yo puedo darle algunas sugerencias o decirle si hay algo que cambiaría porque no me parece" (Asistente técnico, entrevista, fase diagnóstica).

A raíz de estas declaraciones, se reconoce nuevamente la divergencia entre sus respuestas y la práctica educativa, ya que si bien el proceso es desarrollado por el equipo educativo, es realizado en su mayor parte por la educadora, a pesar de que el proceso de evaluación debe ser construido en conjunto con otros agentes educativos, tales como el equipo de aula y los niños y niñas, para favorecer así la recolección de información a través de diversos puntos de vista (García, 2014).

Asimismo, se buscó también obtener información sobre la evaluación de los propios niños y niñas que componen el nivel por 
medio de una encuesta en la fase de planificación, en la que se les preguntó por la percepción que tienen sobre su participación en la evaluación educativa, a lo que sus respuestas fueron:

“¿Qué es la evaluación, miss?" (Niños y niñas, nota de campo, fase de diagnóstico).

“Eso es como cuando decimos si lo hicimos bien o si lo hicimos mal?” (Niños y niñas, nota de campo, fase de diagnóstico).

Con posterioridad a la ejecución de las acciones mencionadas, se les consultó a los participantes, por medio de una encuesta, qué les parecieron estos cambios, y los apoderados señalaron, por ejemplo, que:

"Fue súper enriquecedor para nosotros realizar evaluaciones mientras nuestros hijos desarrollaban sus tareas, ya que podíamos observar instantáneamente cuáles eran sus fortalezas y debilidades, lo cual nos permitía potenciarlas constantemente desde el hogar" (Apoderado 3, nota de campo, fase de reflexión).

Por otro lado, el equipo educativo declaró que:

"Se han observado grandes avances en los aprendizajes de los niños y nińas, sobre todo de quienes estaban considerados dentro del grupo descendido, y eso es gracias a las evaluaciones personalizadas que se realizaron, ya que nos permitieron acompañar de manera oportuna y pertinente según la necesidad" (Equipo de aula, nota de campo, fase de reflexión).

Por su parte, los nińos y niñas comenzaban a verbalizar constantemente los criterios de evaluación que habían establecido, por lo que se podían oír constantemente comentarios tales como:

"Con la evaluación ahora podemos saber qué tenemos que mejorar y siempre que hacemos tareas yo me doy cuenta de lo que necesito seguir practicando" (Niño 1, nota de campo, fase de reflexión). 
Finalmente, culminando con la fase de reflexión, se decidió hacer entrega de encuestas que permitieron evidenciar la percepción de los participantes respecto de la investigación realizada. El equipo educativo respondió la encuesta acompañada de una retroalimentación conversada, destacando el trabajo realizado por la dedicación y minuciosidad al momento de diseñar acciones que generaron cambios, recomendaciones, etc.

"En todo momento, las acciones se llevaron a cabo con respeto, pensando siempre en el beneficio de los niños/as; además, fue súper satisfactorio para los nińos esta investigación, ya que las acciones realizadas permitieron generar acompańamiento y potenciación oportuna, mayor alianza entre la familia y escuela, mejor comunicación con el equipo de aula para distribuir roles y organizarnos en la rutina diaria incorporando la evaluación como un momento establecido. Pero me hubiera gustado que fuera con más tiempo" (Educadora, encuesta, fase de reflexión).

"Considero súper buena la idea de realizar una investigación; siempre hay algunas situaciones que se deben mejorar. No incorporaría ninguna otra acción, ya que las que pensé las dije siempre; sin embargo, sería bueno ver como todo esto se mantiene" (Asistente técnico, encuesta, fase de reflexión).

Según la información recogida mediante las encuestas, se afirma que esta investigación-acción tuvo repercusiones positivas en el nivel educativo; se evidenció que este tipo de investigación es pertinente y enriquece la práctica pedagógica en los niveles de educación parvularia (Guerrero, 2016). Se generaron cambios consistentes que favorecieron tanto al equipo como al grupo de los niños y nińas, demostrando que fue posible reestructurar aspectos de la práctica educativa e implementar un modelo que las invitó a diversificar las estrategias de evaluación y mejorar sus prácticas evaluativas por medio de la reflexión (Elmore, 2010). Esto se logró gracias al trabajo sistemático que se realizó en cada fase de la investigación, en la que se empleó de manera consistente cada observación, nota de campo y encuestas 
que entregaron información relevante para continuar avanzando con nuestro objetivo.

No obstante, se considera relevante destacar primeramente que la autoevaluación desarrollada por lo niños y niñas sorprendió en cuanto a la postura adquirida por ellos, ya que si bien en un comienzo no sabían lo que era ni cómo se desarrollaba, al finalizar el proceso se les pudo ver llevándola a cabo de manera autónoma después de cada instancia de aprendizaje, demostrando que la etapa evolutiva en la que se encontraban les permitió aportar positivamente en este proceso con sus puntos de vista y sus decisiones de mejora. Incluso se observó que comenzó a surgir entre ellos una coevaluación que podría ser llevada a cabo posteriormente con mayor intencionalidad (Basurto et al., 2021).

Por otra parte, logró mejorar el instrumento evaluativo e incluir los registros descriptivos como herramienta de evaluación. De igual manera, se consiguió establecer una instancia permanente para coordinar con anticipación los objetivos diarios a trabajar con el nivel educativo, informando de manera explícita los focos de observación para ese día a los agentes evaluadores, ya que, tal como lo establece García (2014), la evaluación cuenta con el beneficio de poder ser realizada por diferentes agentes y no solo por la educadora de párvulos.

\section{CONCLUSIONES}

Recapitulando la información evidenciada, se corrobora que la evaluación educativa es fundamental en el proceso de enseñanza y aprendizaje, tal como lo establecen las BCEP y MBE-EP, ya que permite obtener la información necesaria para enriquecer el quehacer pedagógico de los educadores. Se ratifica lo planteado también por Díaz y Hernández (2010), respecto de la evaluación como herramienta fundamental dentro del proceso de enseñanza y aprendizaje, que permite integrar mejoras que intencionen aprendizajes significativos. 
Durante el proceso de investigación se logró generar diversas instancias para reflexionar respecto de la evaluación, evidenciando por parte del equipo de aula, los apoderados, las niñas, los niños y la investigadora que la investigación-acción realizada fue un gran aporte, ya que a través de ella se originaron cambios importantes dentro del nivel, lo que mostró logros significativos por medio de las modificaciones. Mejoró tanto el modelo evaluativo como las prácticas evaluativas que tenía el equipo pedagógico, proceso que fue acompañado de reflexiones constantes que permitieron evidenciar su repercusión positiva en la construcción del aprendizaje de los niños y niñas.

Por otro lado, en relación con los objetivos específicos, la investigación permitió diagnosticar las acciones implementadas en el proceso evaluativo del NT2 y su repercusión en la construcción del aprendizaje de niños y niñas; también permitió diseñar e implementar una propuesta evaluativa mejorada junto con el equipo pedagógico, que las condujo a reconocer la importancia de la sistematización de la información relevante que se obtiene durante la práctica pedagógica y lo necesario que es coordinarse para analizar la evaluación realizada por los diversos agentes que participan de ella y en la toma decisiones (García, 2014).

Cabe destacar que, para alcanzar estos resultados, fue un factor sumamente influyente la disposición con la que contó el equipo educativo, quienes evidenciaron una actitud activa y colaboradora a lo largo de la investigación, lo cual permitió dejar en evidencia también la importancia de constituir un equipo de trabajo consistente y aliado que les permita crecer constantemente como profesionales.

Por último, es relevante también explicitar la existencia de limitaciones observadas durante la investigación, así como la incoherencia entre el discurso y el actuar del equipo respecto de la evaluación educativa. Si bien en un comienzo era considerada como un proceso importante, no se observó hasta después de la investigación que existieran espacios establecidos que permitieran recoger, analizar y sistematizar la información pedagógica. Esto explica que en el 
momento inicial el equipo no considerase la necesidad de generar cambios. Al respecto, Tobón (2011) determina que, aunque los docentes en educación inicial reconocen la importancia de evaluar, existe una serie de vacíos o factores influyentes que debilitan esa instancia, tales como: falta de claridad o falta de tiempo para implementarla. Por esta razón, Cáceres et al. (2018) declaran que en, muchas escuelas e instituciones educativas, las y los docentes no poseen los elementos mínimos para establecer las estrategias e instrumentos de evaluación pertinentes a su contexto.

Se logró apreciar también una limitación en la continuidad de la investigación, ya que se propuso implementar esta evaluación en los otros niveles de transición, obteniendo una respuesta negativa por parte de las educadoras de esos niveles.

Finalmente, se considera relevante también destacar la posible ausencia de autocrítica por parte de los educadores, ya que no es común entre ellos analizar sus prácticas pedagógicas para mejorar las acciones que realizan en su rol como docentes; esta parece una gran limitación para incentivar el cambio a nivel de institución educativa. Por ello, se sugieren nuevas investigaciones relacionadas con la reflexión del quehacer educativo y/o el análisis de qué tan factibles son nuestras prácticas pedagógicas para las grandes diversidades de niños y niñas con los que trabajamos anualmente.

\section{REFERENCIAS}

Aguiar, P. (2015). Observación participante: una introducción. Revista San Gregorio, 1(7), 80-89. http://dx.doi.org/10.36097/rsan.v0i0.116

Altimir, D. (2011). Documentar procesos, recoger señales. En R. Sensat (Ed.), Documentar la vida de los niñoslas en la escuela. Red Territorial de Educación Infantil de Cataluña. Octaedro. 
Arrivas, J. (2017). La evaluación de los aprendizajes. Problemas y soluciones. Profesorado. Revista de Currículum y Formación de Profesorado, 21(4), 1138-414. https://recyt.fecyt.es/index.php/ profesorado/article/view/62511

Basurto, T., Moreira, A., Velásquez, N., y Rodríguez, M. (2021). Autoevaluación, coevaluación y heteroevaluación como enfoque innovador en la práctica pedagógica y su efecto en el proceso de enseñanza-aprendizaje. Polo del Conocimiento, 6(3), 828-845. http://dx.doi.org/10.23857/pc.v6i3.2408

Battro, A. M. (2011). Neuroeducación: el cerebro en la escuela. En S. Lipina y M. Sigman (Eds.), La pizarra de Babel. Puentes entre las neurociencias, psicología y educación (pp. 25-70). Libros del Zorzal.

Bericat, E. (1998). La integración de los métodos cuantitativo y cualitativo en la investigación social. Editorial Ariel.

Bonilla, E. y Rodríguez, P. (2005). Más allá del dilema de los métodos. Nomos.

Cáceres, M., Gómez, E. y Zúñiga, M. (2018). El papel del docente en la evaluación del aprendizaje. Revista Conrado, 14(63), 196207. http://conrado.ucf.edu.cu/index.php/conrado

Denzin, N. y Lincoln, Y. (2005). The Sage Handbook of Qualitative Research. Sage.

Díaz, F. y Hernández, G. (2010) Estrategias docentes para un aprendizaje significativo: una interpretación constructivista (2a ed.). McGraw-Hill.

Elliott, J. (1993). El cambio educativo desde la investigación-acción. Ediciones Morata.

Elmore, R. (2010). Mejorando la escuela desde la sala de clases. Área de Educación Fundación Chile. 
Garbin, C., Garbin, A., Saliba, N., Zina, L. y Gonzales, P. (2007). El consentimiento informado en la Clínica Odontológica. Acta Odontológica Venezolana, 45(1), 1-10. https://www.actaodontologica. com/ediciones/2007/1/art-7/

García, J. (2014). Fundamentos pedagógicos de la evaluación. Síntesis.

García, T., García, L., González, R., Carvalho, J. y Catarreira, S. (2016). Revisión metodológica de la triangulación como estrategia de investigación. Atas-Investigação Qualitativa em Ciências Sociais, 3. https://proceedings.ciaiq.org/index.php/ciaiq2016/ article/view/1009

Garza, E. de la (2004). La evaluación educativa. Revista Mexicana de Investigación Educativa, 23(9), 807-816. http://www.redalyc. org/articulo.oa?id=14002302

Gómez, R. y Seda, S. (2008). Creencias de las educadoras acerca de la evaluación de sus alumnos preescolares: un estudio de caso. Perfiles Educativos, 30(119), 33-54. http://www.scielo.org.mx/scielo. php?script=sci_arttext\&pid=S0185-26982008000100003\&lng= es\&nrm=i\&tlng=es

Guerrero, A. (2016). La investigación cualitativa. INNOVA Research Journal, 1(2), 1-9. https://doi.org/10.33890/innova.v1.n2.2016.7

Hoyuelos, A. y Riera M. (2015). Complejidad y relaciones en educación infantil. Octaedro.

Ibáñez, R. (2020). Los procesos de evaluación y toma de decisiones en el desarrollo y aprendizaje de los párvulos en jardines infantiles de la Junta Nacional de Jardines Infantiles de la Región Metropolitana, Chile. Pensamiento Educativo, 57(1), 1-20. http:// dx.doi.org/10.7764/pel.57.1.2020.3

Jensen, E. (2004). Cerebro y aprendizaje. Competencias e implicaciones educativas. Narcea Ediciones.

Latorre, A. (2005). La investigación-acción: conocer y cambiar la práctica educativa. Graó. 
Letelier, S., Gomez, G. y Vizcarra, T. (2020). Estudio sobre el sistema de calidad para la Educación Parvularia de Chile. En XXVI Jornadas de Investigación en Psicodidáctica, pp. 127-139. https:// dialnet.unirioja.es/servlet/articulo?codigo=7432599

Losada, H. y Iturbide, Á. (2011). Aplicación de la investigación social a la evaluación y su relación con la taxonomía de Bloom. Indagatio Didáctica, 3(3). 1-18. https://doi.org/10.34624/id.v3i3.4534

Malaguzzi, L. (2001). La educación infantil en Reggio Emilia. Octaedro.

Martínez, S. y Rochera, J. (2010). Las prácticas de evaluación de competencias en la educación preescolar mexicana a partir de la reforma curricular: Análisis desde un modelo socioconstructivista y situado. Revista Mexicana de Investigación Educativa, 15(47), 1025-1050. http://www.redalyc.org/articulo.oa?id=14015564003

Ministerio de Educación (2016). Plan de Aseguramiento de la Calidad Escolar 2016-2019. Santiago, Chile: MINEDUC.

Muñoz, M. (2011). Estudio aplicativo sobre comprensión lectora en Estudiantes de NT2 con alto indice de vulnerabilidad de la comuna de Talca. [tesis doctoral]. Universidad Católica del Maule. http:// repositorio.ucm.cl/handle/ucm/3282

Norambuena, K., Palma, C., Pinochet, C., Quinteros, K., Valenzuela, J. y Valderrama, T. (2012). Descripción de competencias de estudiantes del $2^{\circ}$ nivel transición y nivel básico en sectores de Matemáticas y Lenguaje y comunicación de establecimientos municipales de la comuna de Talca [tesis doctoral]. Universidad Católica del Maule. http://repositorio.ucm.cl/handle/ucm/2931

Paniagua, G. y Palacios, J. (2005) Educación Infantil: Respuesta educativa a la diversidad. Alianza Editorial.

Rivera, A. y García, L. (2016). La evaluación para el aprendizaje en la educación infantil o parvularia. Infancia, Educación y Aprendizaje (IEYA), 2(2), 106-117. https://doi.org/10.22370/ieya.2016.2.2.596 
Rojas, F. (2012). La investigación acción y la práctica docente. Cuaderno de Educación, (41), 1-8. https://repositorio.uahurtado. cl/handle/11242/6495

Santamaría, S. (2013). Paradigmas de investigación educativa: de las leyes subyacentes a la modernidad reflexiva. Entelequia: Revista Interdisciplinar, (16), 91-102. https://dialnet.unirioja.es/servlet/ articulo? codigo $=4768090$

Subsecretaría de Educación Parvularia (2018). Bases Curriculares de Educación Parvularia. Gobierno de Chile. Ministerio de Educación. https://parvularia.mineduc.cl/wp-content/uploads/ sites/34/2018/03/Bases_Curriculares_Ed_Parvularia_2018.pdf

Subsecretaría de Educación Parvularia (2019). Marco para la Buena Enseñanza de Educación Parvularia. Gobierno de Chile. Ministerio de Educación. https://parvularia.mineduc.cl/wp-content/uploads/ sites/34/2019/08/MBE_EP-Final.pdf

Subsecretaría de Educación Parvularia (2020). Orientaciones para Planificar y evaluar a distancia en el nivel de educación parvularia. Un desafio para los equipos pedagógicos en contexto COVID-19. Gobierno de Chile. Ministerio de Educación. https://parvularia. mineduc.cl/orientaciones-planificar-evaluar/

Tobón, S. (2011). Evaluación de las competencias en la Educación Básica. Santillana.

Tójar, J. C. y Serrano, J. (2000). Ética e investigación educativa. Relieve, 6(2), 1-6. http://www.uv.es/RELIEVE/v6n2/ RELIEVEv6n2_2.htm

Vera, A. y Villalón, M. (2005). La triangulación entre métodos cuantitativos y cualitativos en el proceso de investigación. Ciencia y Trabajo, 7(16), 85-87. http://www.uprh.edu/elopez/13\%20 Triangulacion.pdf 